\title{
PERCEPÇÃO DO ATENDIMENTO ODONTOLÓGICO: COMPARAÇÕES ENTRE GRUPOS DE GESTANTES ADULTAS E ADOLESCENTES
}

\author{
PERCEPTION OF DENTAL CARE: COMPARISON BETWEEN PREGNANT \\ ADULTS WOMEN AND ADOLESCENTS
}

\section{Constanza Marín ${ }^{a^{*}}$, Célio Afonso Rieg Maçaneiro ${ }^{b^{*}}$, Elisabete Rabaldo Bottan ${ }^{\mathrm{c}^{*}}$, Felipe Vavassori ${ }^{\mathrm{d**}}$}

\author{
acconstanzamarin4@gmail.com, bcelioafonsorm@gmail.com, cerabaldo@univali.br, dvavassori@gmail.com \\ *Universidade do Vale do Itajaí - Itajaí (SC), Brasil \\ **Universidade da Região de Joinville - Joinville (SC), Brasil
}

Data de recebimento do artigo: 04/12/2014

Data de aceite do artigo: 28/05/2015

\section{RESUMO}

Introdução: Informações sobre o conhecimento das gestantes sobre saúde bucal permitem um planejamento adequado de ações de promoção de saúde durante a gravidez. Objetivo: Conhecer as percepções de gestantes sobre tratamento odontológico durante a gravidez e identificar se há influência da faixa etária na manifestação dessas percepçóes. Métodos: Pesquisa descritiva, transversal. Foram constituídos aleatoriamente dois grupos: adultas e adolescentes. A coleta de dados ocorreu mediante questionário autoaplicável. Os dados foram analisados com base na estatística descritiva; foi aplicado o teste do qui-quadrado ( $<<0,01)$. Resultados: Participaram da pesquisa 112 gestantes adultas e 80 adolescentes. A idade média das adultas foi de 27,43 anos e entre as adolescentes foi 16,6 anos. Em ambos os grupos, a maioria possuía ensino médio. A renda mensal mais relatada, nos dois grupos, foi de até três salários mínimos. $\mathrm{O}$ temor à consulta odontológica foi revelado por $29,7 \%$, e $34,4 \%$ não efetuam consulta odontológica nessa fase. A maior frequência de consultas odontológicas e da ausência de medo da consulta durante a gravidez ocorreu no grupo de adolescentes, muito embora tal diferença não tenha sido estatisticamente significativa. $\mathrm{O}$ temor mais relatado pelas participantes dos dois grupos foi anestesia. As adolescentes evidenciaram um melhor conhecimento em três das quatro questóes do campo cognitivo em comparação ao grupo de gestantes adultas, com diferença estatisticamente significativa. Conclusão: $\mathrm{O}$ temor ao tratamento odontológico durante a gravidez foi identificado em baixa frequência na amostra. A faixa etária exerceu influência na maioria das respostas do campo cognitivo e para dois dos temores citados.

Palavras-chave: Gestantes; saúde bucal; promoção da saúde.

\section{ABSTRACT}

Introduction: Information on the knowledge of pregnant women about oral health enables a proper planning of health promotion activities during pregnancy. Objective: To know the perceptions of pregnant women about dental treatment during pregnancy and identify if there is influence of age on the manifestation of these perceptions. Methods: Transversal descriptive research. Two groups were randomly constituted: adult pregnant and pregnant adolescents. The instrument for data collection was a self-applicable questionnaire. Data were analyzed using descriptive statistics; the chi-square test was applied $(\mathrm{p}<0.01)$. Results: A total of 112 pregnant adults and 80 adolescents participated in this study. The average age of group 1 was 27.43 years and the group 2 was 16.6 years. In both groups the majority owned high school. The fear of dental appointment was revealed by $29.7 \%$ of the surveyed and $34.4 \%$ do not make dental appointment at this stage. The most reported monthly income in both groups was up to three minimum wages. Adolescents reported the higher frequency of dental appointments and not to be afraid of dental appointments during pregnancy, although this difference was not statistically significant. The most reported fear of the participants of both groups was anesthesia. Adolescents showed better knowledge; in three 
of the four issues of the cognitive field, such difference is statistically significant. Conclusion: Fears about the dental treatment during pregnancy has been identified at low frequency in the sample. Age exerted influence on most of the answers of the cognitive field and in two of the mentioned fears.

Keywords: Pregnant women; oral health; health promotion.

\section{Introdução}

A consulta odontológica é muito importante durante a gestação, no entanto, existe falta de conhecimento sobre a sua necessidade, bem como sobre a segurança do tratamento odontológico durante a gestação. A literatura atual é consistente e recomenda que, durante o período gestacional, a mulher seja examinada e avaliada quanto ao risco de doenças bucais, aconselhada sobre cuidados para com a higiene e encaminhada para o tratamento quando necessário. Além do mais, o tratamento periodontal, radiografias, restauraçóes e extraçóes podem ser realizados com segurança ${ }^{1-8}$.

$\mathrm{O}$ conhecimento das gestantes sobre a atenção à saúde bucal durante a gestaçáo é de suma importância para o desenvolvimento de hábitos saudáveis. De acordo com as atuais Diretrizes da Política Nacional de Saúde Bucal, a educação em saúde bucal deverá ser incluída transversalmente no atendimento das gestantes de forma que a mulher possa atuar como multiplicadora de informaçóes e promotora de saúde bucal. Para isto, ela precisa ter esclarecimentos precisos e estar consciente do seu papel na aquisição e manutenção de hábitos positivos de saúde no ambiente familiar ${ }^{2,5,6,8-10}$.

A atenção odontológica durante a gestação deve ser vista como uma possibilidade para estabelecimento, incorporação e mudanças de hábitos, pois nesse período, geralmente, a mulher manifesta inúmeras dúvidas sobre as quais necessita de informações adequadas. Acreditase que, se as gestantes estiverem bem orientadas, apresentarão melhorias no autocuidado em relação à sua saúde bucal, bem como de seus filhos ${ }^{1-10}$.

O impacto das ações de promoção de saúde é superior quando se realiza preliminarmente um levantamento de quais são os saberes dos sujeitos, planejando as intervençôes com base nos seus resultados. Estes procedimentos favorecem a motivação e a adesão aos processos de cuidados para com a saúde. Logo, o primeiro momento de um programa de atenção à saúde da gestante deve ser investigar a sua percepção sobre os autocuidados para com a sua saúde bucal ${ }^{8,10}$. Para Finkler et al. ${ }^{10}$, compreender os saberes relacionados à saúde bucal sob a perspectiva dos próprios sujeitos é importante possibilita o planejamento de açóes educativas mais democráticas, delineadas a partir da própria populaçáo a que se destinam, portanto, favorecendo a sua participação no controle do processo saúde-doença.
Por se tratar de um tema de grande importância em saúde pública e que exerce influência direta nos cuidados relacionados à gestação, faz-se necessário identificar os mitos, tabus, medos e anseios que fazem parte do ideário das gestantes, visto que a atenção odontológica nessa fase é carregada de preconceitos que podem dificultar a adesão das gestantes a esse tipo de cuidados ${ }^{1-13}$.

Assim, tendo em vista a ênfase que os cursos de odontologia das universidades comunitárias de Santa Catarina vêm dando à integração ensino-serviço, em especial na atenção básica, esta pesquisa foi dimensionada no sentido de se obter subsídios à reorientação dos programas educativos direcionados às gestantes, que vêm sendo ofertados na área de abrangência das instituiçóes. Considerando tais aspectos, o objetivo desta pesquisa foi conhecer as percepçóes de mulheres grávidas sobre os procedimentos odontológicos durante a gravidez e identificar se há influência da faixa etária na sua manifestação.

\section{Materiais e métodos}

Esta investigação caracterizou-se como um estudo descritivo, do tipo transversal, mediante levantamento de dados primários. O projeto de pesquisa foi submetido e aprovado pelo Comitê de Ética em Pesquisa da Univali sob o no 113 e da Univille sob o no 240.844 .

Foram constituídos aleatoriamente dois grupos, sendo um com gestantes adultas (Grupo 1) e outro com adolescentes (Grupo 2). Nos dois grupos foram identificadas as primigestas e não primigestas.

Os critérios para a inclusão no estudo foram: ser gestante com idade superior a 18 anos, para o grupo de adultas, e inferior a 18 anos para o grupo de adolescentes; estar em atendimento pré-natal; e aceitar por livre e espontânea vontade participar da pesquisa e assinar o Termo de Consentimento Livre Esclarecido.

A coleta de dados ocorreu no período de março a junho de 2013, em dois ambientes: Unidade Básica de Saúde e Hospital, localizados em duas cidades do litoral norte de Santa Catarina.

Utilizou-se como instrumento de coleta de dados um questionário composto por onze questóes do tipo fechado, distribuídas em dois campos. O primeiro campo referia-se à caracterização da gestante (idade, escolaridade, renda, número de gestaçóes). O segundo campo 
continha questóes sobre conhecimento e condutas em relação ao atendimento odontológico durante a gravidez.

Os dados foram analisados com base na estatística descritiva, mediante cálculo da distribuição da frequência relativa, e posteriormente foi aplicado o teste não paramétrico do qui-quadrado, considerando como diferença significativa aquelas definidas por um "p" crítico igual ou menor que 0,01 .

\section{Resultados}

Participaram da pesquisa um total de 192 gestantes, sendo 112 do grupo 1 (adultas) e 80 do grupo 2 (adolescentes). Sobre o número de gestaçóes, no grupo das adultas, 32\% ( $\mathrm{n}=36)$ relataram estar em sua primeira gravidez e $68 \%(n=76)$ já haviam passado por outra gestação. Entre as adolescentes, $89 \%(\mathrm{n}=71)$ estavam na sua primeira gravidez e $11 \%(n=9)$ náo eram primigestas.

As integrantes do grupo 1 tinham idades entre 19 e 39 anos, sendo a idade média de 27,43 anos. No grupo de gestantes adolescentes, as idades variaram de 14 a 17 anos, com idade média de 16,6 anos. Em ambos os grupos a maioria possuía ensino médio (completo ou incompleto). A renda mensal mais relatada nos dois grupos foi de até três salários mínimos.

Os resultados das questóes relativas às condutas quanto à consulta odontológica durante a gravidez apontam que $29,7 \% \quad(\mathrm{n}=57)$ do total das pesquisadas (192) revelaram temor à consulta odontológica, e $34,4 \%(n=66)$ afirmaram não efetuar acompanhamento odontológico nessa fase de suas vidas. Na Tabela 1 podem ser observados os resultados relativos a essas condutas segundo os grupos constituídos.

Tabela 1: Condutas quanto à consulta odontológica durante a gravidez.

\begin{tabular}{lccccc}
\hline Pergunta & \multicolumn{2}{c}{ Grupo 1 } & \multicolumn{2}{c}{ Grupo 2 } & $\begin{array}{c}\text { Valor } \\
\text { p }\end{array}$ \\
\hline & Sim & Não & Sim & Não & \\
$\begin{array}{l}\text { Visita o } \\
\text { dentista duran- } \\
\text { te a gestaçáo? }\end{array}$ & 67 & 45 & 59 & 21 & 0,04 \\
$\begin{array}{l}\text { Tem medo de } \\
\text { consultar o } \\
\text { dentista duran- } \\
\text { te a gestaçáo? }\end{array}$ & 35 & $(40 \%)$ & $(74 \%)$ & $(26 \%)$ & \\
\hline
\end{tabular}

Para estas duas questóes, quando se procedeu à análise de primigestas versus não primigestas, somente no grupo 1 (adultas), para o quesito "ter medo de efetivar consulta odontológica durante a gravidez", é que se identificou uma diferença significativa $(p=0,001)$, sendo as primigestas menos temerosas. No entanto, a análise entre primigestas adolescentes e primigestas adultas não apresentou diferença estatística.

As gestantes que relataram ter medo da consulta odontológica durante a gravidez, ao serem indagadas sobre quais seriam as situaçóes que mais lhes suscitavam temor, citaram: tomadas radiográficas, ingestão de remédios receitados pelo cirurgião-dentista, procedimentos durante o tratamento e aplicaçáo do anestésico local. $\mathrm{O}$ temor mais relatado foi referente à anestesia (Gráfico 1).

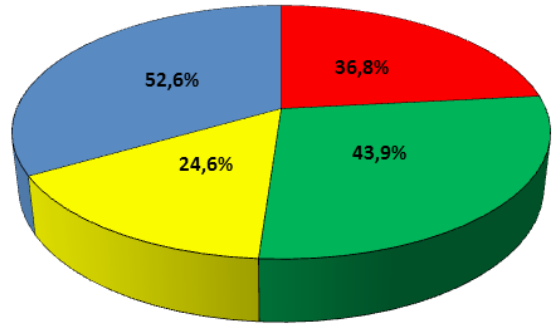

$\square \mathrm{RX} \square$ Medicação $\square$ Procedimentos clínicos diversos $\square$ Anestesia

Gráfico 1: Frequência das situações consideradas desencadeadoras de medo.

Quando se analisa a frequência das situaçóes relacionadas ao tratamento odontológico durante a gestação consideradas como desencadeadoras de medo, segundo os grupos pesquisados, observa-se que o grupo 1 (adultas) foi o que obteve as frequências mais altas para a categoria "sim", que designava ter medo. Também se confirma que o temor à anestesia é o mais frequente para ambos os grupos (Tabela 2).

Tabela 2: Situações relacionadas ao tratamento odontológico consideradas desencadeadoras de medo, segundo os grupos pesquisados.

\begin{tabular}{lccccc}
\hline & \multicolumn{4}{c}{ Grupo 1 } & \multicolumn{2}{c}{ Grupo 2 } \\
\hline Fatores & $\begin{array}{l}\text { Sim } \\
(\%)\end{array}$ & $\begin{array}{c}\text { Não } \\
(\%)\end{array}$ & $\begin{array}{c}\text { Sim } \\
(\%)\end{array}$ & $\begin{array}{c}\text { Não } \\
(\%)\end{array}$ & $\begin{array}{c}\text { Valor } \\
\text { p }\end{array}$ \\
Anestesia & 65,7 & 34,3 & 31,8 & 68,2 & $0,01^{*}$ \\
Medicaçáo & 62,9 & 37,1 & 13,6 & 86,4 & $0,00^{*}$ \\
Raio X & 48,6 & 51,4 & 18,2 & 81,8 & 0,02 \\
$\begin{array}{l}\text { Procedimentos clínicos } \\
\text { diversos }\end{array}$ & 34,3 & 65,7 & 9,1 & 90,9 & 0,03 \\
\hline
\end{tabular}

*Valores estatisticamente significativos.

Os resultados relativos às questóes de domínio cognitivo, segundo os grupos, estáo dispostos na Tabela 3. 
Tabela 3: Respostas referentes ao domínio cognitivo, segundo os grupos pesquisados.

\begin{tabular}{cccccc}
\hline & \multicolumn{2}{c}{ Grupo 1 } & \multicolumn{2}{c}{ Grupo 2 } & $\begin{array}{c}\text { Valor } \\
\text { p }\end{array}$ \\
\hline Pergunta & Sim & Não & Sim & Não & \\
$\begin{array}{c}\text { Relaçáo DP com } \\
\text { baixo peso do bebê }\end{array}$ & 57 & 55 & 28 & 50 & 0,04 \\
$\begin{array}{c}\text { Gravidez enfraquece } \\
\text { os dentes }\end{array}$ & 60 & 52 & 26 & 52 & $0,00^{*}$ \\
$\begin{array}{c}\text { Cárie pode ser trans- } \\
\text { mitida ao bebê } \\
\text { Tratamento }\end{array}$ & $(54 \%)$ & $(46 \%)$ & $(33 \%)$ & $(67 \%)$ & \\
$\begin{array}{c}\text { odontológico pode } \\
\text { ser realizado }\end{array}$ & 86 & $(57 \%)$ & $(74 \%)$ & $(26 \%)$ & $0,00^{*}$ \\
durante a gestaçáo & $(77 \%)$ & $(23 \%)$ & $(90 \%)$ & $(10 \%)$ & $0,01^{*}$ \\
\hline
\end{tabular}

$\mathrm{DP}=$ Doença Periodontal

* Valores estatisticamente significativos.

\section{Discussão}

A gestação é um período fisiológico complexo, pois, além das alteraçóes físicas e emocionais, existem crenças e mitos envolvendo o binômio mãe-filho. Mitos como a relaçáo entre a gravidez e a perda dos dentes, ou de que a consulta odontológica é prejudicial durante a gravidez, por muitos anos estiveram plenamente enraizados na cultura popular. Tềm se perpetuado passando por geraçóes e, mesmo na atualidade, ainda persistem para muitas pessoas ${ }^{4,5,7,10-17}$.

Apesar da existência desses mitos, há que se considerar que, durante a gestação, a mulher está mais receptiva à aquisição de novos conhecimentos e à mudança de hábitos que se relacionem à saúde de seu bebê. A literatura tem demonstrado que mães bem informadas e motivadas cuidam melhor de sua da saúde bucal e de seus filhos ${ }^{2,4,5,9,12,13}$. No entanto, diversas barreiras interferem na busca pelo atendimento odontológico durante a gravidez, entre as quais podemos citar os aspectos financeiros e a pouca oferta de serviços odontológicos na rede pública. Aliada a estes fatores, a falta de conhecimento sobre a necessidade de atendimento odontológico e o medo de certos procedimentos clínico-odontológicos durante a gravidez dificultam significativamente o acesso aos cuidados para com a saúde bucal da gestante $e^{3,14,17}$.

As práticas de atenção à gestante nos serviços de saúde, apesar de inúmeros esforços dispendidos por diferentes setores, ainda são limitadas no que diz respeito à saúde bucal e ao tratamento odontológico na gravidez. O pré-natal odontológico é de extrema importância e não pode ser negligenciado. Deve contemplar, entre outros procedimentos, palestras de educação em saúde bucal, anamnese detalhada e orientaçóes qualificadas que favoreçam a ruptura de preconceitos da paciente $e^{2,7,15-17}$.
Conforme preconizado pelo Ministério da Saúde ${ }^{18}$, a equipe de saúde bucal deve ouvir as gestantes sobre problemas, crenças e tabus, e os profissionais da saúde devem compreender que os saberes do senso comum e as crenças transmitidas de uma geração a outra são influenciados pelos processos sociais. Portanto, devem respeitar a forma de pensar das gestantes, mas desmitificar as crenças que constituem obstáculos à adoção de hábitos de vida saudáveis ${ }^{10,19}$.

Atento a esses aspectos, o governo brasileiro vem direcionando mais esforços à saúde materno-infantil. Em 2011, o Ministério da Saúde instituiu no âmbito do Sistema Único de Saúde a estratégia Rede Cegonha, normatizada pela Portaria no 1.459 /GM/MS, de 24 de junho de 2011, e, posteriormente, alterada pela Portaria $n^{\circ}$ 2.351, de 5 de outubro de 2011. A estratégia tem como objetivo ampliar o acesso e melhorar a qualidade da atençáo pré-natal, a assistência ao parto e ao puerpério e a assistência à criança com até 24 meses de vida, por meio da vinculação da gestante à unidade de referência ${ }^{20}$.

É importante destacar que, entre as gestantes que integraram esta pesquisa, mais da metade $(65,62 \%)$ afirmou que costuma efetivar consulta odontológica durante a gestação, no entanto, mulheres de ambos os grupos constituídos (adultas e adolescentes) afirmaram ter medo da consulta odontológica durante a gestaçáo e evitar efetivá-la nessa fase de suas vidas. Este comportamento é apontado em inúmeros estudos realizados em diferentes contextos socioculturais ${ }^{1-16,17,19,21-23}$.

A informação é fator chave que pode interferir na decisão de procurar pelo atendimento odontológico durante a gestação ${ }^{1}$. As gestantes precisam ser orientadas e estimuladas a efetivarem exame odontológico pelo menos uma vez durante a gravidez. Nesta pesquisa, as adultas, em comparação às adolescentes, foram as que menos realizaram consulta odontológica e as que mais admitiram ter medo de efetivá-la durante a gravidez. É possível que tal comportamento se deva ao fato de que mães mais jovens incorporam hábitos saudáveis mais facilmente, são mais receptivas às informações sobre saúde bucal e participativas em reuniōes e palestras educativas $^{12,21,22}$. Pode-se, ainda, considerar que entre as gestantes mais jovens, a experiência de atendimento odontológico tenha sido mais positiva devido ao fato de que, atualmente, a Atenção Básica está orientada em ciclos de vida, de modo que essas adolescentes podem ter experimentado uma odontologia de cunho mais preventivo do que curativo, o que significa dizer que, provavelmente, tenham tido menos experiências negativas do que as adultas.

A existência de crenças e mitos populares relacionados à saúde bucal durante a gravidez influi significativamente nas atitudes das mulheres e, por conseguinte, na qualidade de suas vidas e de seus filhos. Portanto é 
de suma importância oportunizar açóes que favoreçam a mudança de conduta das grávidas com o objetivo de melhorar sua condição de saúde $2,5,6,9,11,13$. Dentre as diferentes crenças envolvendo a gestaçáo, a que se refere ao enfraquecimento dos dentes da grávida em virtude da perda de cálcio da mãe para o feto ainda é muito evocada pelas gestantes ${ }^{2,715}$. Neste estudo, as gestantes adultas foram as que mais demonstraram acreditar que a gravidez enfraquece os dentes da mãe, sendo essa diferença considerada estatisticamente significativa. Este resultado reforça a hipótese de que as mais jovens são mais receptivas aos programas de educação em saúde bucal para gestantes ${ }^{12,21,22}$.

Quando se questionou sobre o medo em relação a diferentes procedimentos clínico-odontológicos $\mathrm{du}$ rante a gravidez, identificou-se que para os dois grupos (adultas e adolescentes) a anestesia é o que mais causa temor. Conforme registros na literatura, muitas grávidas acreditam que a anestesia é um procedimento perigoso que pode acarretar consequências maléficas para si e para o feto ${ }^{7,11,13,15-17,21-23}$. Contudo, atualmente, a utilização de anestésicos é considerada segura, desde que o profissional tenha conhecimento sobre a quantidade da droga a ser administrada, técnica anestésica, ausência/ presença de vasoconstritor e efeitos citotóxicos. A solução anestésica local que apresenta maior segurança em gestantes é a lidocaína 2\% com epinefrina 1:100.000, respeitando-se o limite máximo de dois tubetes anestésicos $(3,6 \mathrm{ml})$ por sessão, procedendo sempre uma lenta injeção da solução $7,15,17,22,23$.

Um grande número de procedimentos odontológicos pode ser realizado durante a gravidez. Nesse aspecto, a maioria das mulheres pesquisadas, nos dois grupos, afirmou que tratamento odontológico pode ser efetivado durante a gestação. No entanto, temos que ressaltar que, muito embora um número expressivo tenha se posicionado assim, encontrou-se um percentual de mulheres que manifestaram temor/preocupação. Por isso, é fundamental que os profissionais da área da saúde reforcem a disseminação de informaçóes sobre os cuidados para com a saúde bucal da gestante.

Deve-se estar alerta para o fato de que, por diversos fatore, as mulheres grávidas estão muito mais suscetíveis à inflamação gengival. Por isso, é importante saberem que as alteraçôes gengivais são limitadas e reversíveis se houver o acompanhamento de um profissional associado à manutençấo de uma adequada higiene bu$\mathrm{cal}^{2,3,7,16-17,23-27}$. A possível associação entre doença periodontal e resultados adversos para o feto enfatiza a importância do atendimento odontológico durante a gestação.

Tem sido relatado na literatura ${ }^{2,3,17,19,24-27}$ que a doença periodontal pode aumentar o risco de parto pré-termo e/ou de baixo peso do bebê ao nascer. Entre as participantes deste estudo, verificou-se pouco expressiva a frequência de mulheres, especialmente no grupo de adolescentes, que demonstraram conhecer a associação entre doença periodontal e complicaçóes para o bebê. A constataçáo reforça a necessidade de serem informadas de que a periodontite pode estar relacionada com nascimentos de baixo peso e que mães com elevados níveis de bactérias cariogênicas podem levar ao aumento de cáries no filho ${ }^{23,24}$

Tratando-se de atenção odontológica à gestante, o cirurgiáo-dentista deve observar cuidados essenciais, tais como: planejar sessóes curtas, adequar a posição da cadeira e evitar consultas matinais, já que neste período a gestante tem mais ânsia de vômito e risco de hipoglicemia ${ }^{7}$. Além do mais, procedimentos clínicos, como diagnóstico, radiografias, tratamento periodontal, anestesia, restauraçóes e exodontias são seguros e podem ser realizados durante a gestaçáo, preferencialmente no segundo trimestre. Quanto ao uso de medicamentos, antibióticos (como penicilina) e analgésicos (como o paracetamol) podem ser prescritos nessa fase de vida da mulher sem que ocorram prejuízos ao feto. Não é recomendado o uso de anti-inflamatórios não esteroidais; quando houver necessidade, devem ser administrados nas menores doses e suspensos oito semanas antes da data prevista para o parto ${ }^{17,19}$.

É necessário, portanto, o estabelecimento de aproximaçóes entre as políticas de atençáo à gestante, mediante o desenvolvimento de diretrizes e protocolos que possam servir de guia comum e propiciar o diálogo entre os profissionais da área da saúde e destes com as gestantes. $\mathrm{O}$ trabalho multiprofissional no cuidado da saúde da gestante corrobora para a melhoria do nível de informação e de conhecimentos destas mulheres o que favorece a superação de resistências, medos, crenças e representaçōes sobre atenção odontológica que funcionam como obstáculos à procura e à adesão à consulta odontológica. Os diversos profissionais de saúde devem se articular nos serviços de assistência pré-natal para que o princípio da integralidade seja efetivado em seus diversos sentidos, tornando o processo de assistência pré-natal humanizado e de qualidade ${ }^{10,14-16}$. Nesse sentido, a estratégia Rede Cegonha, bem como o programa de Saúde da Família são importantes iniciativas que, sem dúvida, ampliarão a oferta de serviços de atenção odontológica à gestante e à criança após o seu nascimento. $\mathrm{O}$ cuidado pré-natal deve associar abordagem clínica, preventiva e educativa, visando ao alcance de hábitos favoráveis à saúde bucal da mãe e do bebê.

Investigaçóes relacionadas à saúde bucal da gestante são necessárias. É preciso avaliar continuamente o padrão de utilizaçáo dos serviços odontológicos entre as mulheres grávidas e determinar quais são as barreiras à procura de tratamento durante a gravidez $z^{3,10,21}$. Nesse 
sentido, este estudo, apesar da limitação no que tange à amostra, se constitui numa contribuição reflexiva para os profissionais da área da saúde. Por se tratar de uma amostra de conveniência, envolvendo uma unidade básica de saúde e um hospital, em que o número de participantes não foi tấo expressivo, seus resultados não podem ser generalizados para toda a população de mulheres grávidas da região estudada.

\section{Conclusão}

De acordo com a metodologia adotada neste estudo, concluiu-se que gestantes adolescentes são menos temerosas à consulta odontológica durante a gravidez, bem como aos diferentes procedimentos clínico-odontológicos, quando comparadas às gestantes adultas. A anestesia e o uso de medicação no período gestacional foram os temores mais citados pelas pesquisadas, no entanto, no grupo de adolescentes a frequência foi bem inferior àquela obtida no grupo das adultas. Tal diferença foi estatisticamente significativa.

A frequência da visita ao dentista durante a gravidez é maior entre as gestantes adolescentes; no entanto, esta diferença não foi estatisticamente significativa.

Para as questóes sobre enfraquecimento dos dentes maternos durante a gravidez, transmissibilidade da cárie e possibilidade de efetivação de tratamento odontológico durante a gestação, a maioria das gestantes adolescentes emitiu respostas corretas, quando comparadas às gestantes adultas, sendo essa diferença estatisticamente significativa.

Muito embora o comportamento e o conhecimento manifestados pelas gestantes adolescentes tenham sido mais positivos em comparação ao grupo de gestantes adultas, há que se destacar que em ambos os grupos ainda há uma parcela de mulheres que denotou desinformação quanto ao atendimento odontológico durante a gravidez.

É preciso, portanto, (re)planejar programas educativo-preventivos que ressaltem os benefícios trazidos pelo pré-natal odontológico. Ações de promoção da saúde devem ser utilizadas como forma de disseminação de conhecimento e empoderamento das gestantes. Indubitavelmente, a literatura pertinente destaca, entre essas ações, que o processo educativo numa dimensão dialógica, de aproximação dos saberes (popular e científico), a médio e longo prazo, tem um impacto altamente positivo na qualidade de vida da gestante, o qual se reflete no âmbito familiar.

\section{Agradecimento}

Ao Fundo de Apoio à Pesquisa da Universidade do Vale do Itajaí.

\section{Referências}

1. Ojeda JC. A literature review on social and economic factors related to access to dental care for pregnant women. The Journal of Dentist. 2013;1(1):24-34.

2. Reis DM, Pitta DR, Ferreira HMB, de Jesus MLP, de Soares MEL, Soares MG. Educação em saúde como estratégia de prevenção de saúde em gestantes. Cienc saúde coletiva. 2010;15(1)269-76.

3. Al-Swuailem AS, Al-Jamal FS, Helmi MF. Treatment perception and utilization of dental services during pregnancy among sampled women in Riyadh, Saudi Arabia. King Saud University jornal of dental sciences. 2014;5(2):123-9.

4. Núñez J, Moya P, Monsalves MJ, Landaeta MS. Nivel de conocimiento de salud oral y utilización de GES odontológico en puérperas atendidas en una clínica privada, Santiago, Chile. Int. J. Odontostomat. 2013;7(1): 39-46.

5. Codato LAB, Nakama L, Cordoni Junior L, Higasi MS. Atenção odontológica à gestante: papel dos profissionais de saúde. Ciênc saúde coletiva. 2011;16(4):2297-2301.

6. Mesquita BS, Meneses IHC, Pessoa TRRF, Farias IAP. Aspectos norteadores da atenção à saúde bucal de gestantes da estratégia saúde da família de João Pessoa, Brasil. ROBRAC. 2013;21(60):45-9.

7. Bastiani C, Cota ALS, Provenzano MGA, Fracasso MLC, Honorio HM, Rios D. Conhecimento das gestantes sobre alteraçóes bucais e tratamento odontológico durante a gravidez. Odontol Clin Cient. 2010;9(2):155-60.

8. Paulino HH, Souza P, Codato LAB, Muraguchi EMO, Higasi MS, Casaroto P, et al. Grupo de gestantes: uma estratégia de intervenção do PET-Saúde da Família. Rev da ABENO. 2013;13(2):76-81.

9. Dobarganes Coca AM, Lima Álvarez M, López Larquin $\mathrm{N}$, Pérez Cedrón RA, González Vale L. Intervención educativa en salud bucal para gestantes. AMC. 2011;15(3):528-41.

10. Finkler M, Oleiniski DMB, Ramos FRS. Saúde bucal materno-infantil: um estudo de representaçôes sociais com gestantes. Texto Contexto Enferm. 2004;13(3):360-8.

11. Amorim BF, Costa JF, Costa EL. Percepção de primigestas adolescentes sobre saúde bucal. Rev de Pesquisa em Saúde. 2011;12(2):13-7.

12. Garbin CAS, Sumida DH, Santos RR, Chehoud KA, Moimaz SAS. Saúde coletiva: promoção de saúde bucal na gravidez. Rev Odontol UNESP. 2011;40(4):161-5.

13. Nogueira LT, Valsecki Junior A, Martins CR, Rosell FL, Silva SRC. Retardo na procura do tratamento odontológico e percepção da saúde bucal em mulheres grávidas. Odontol Clín Cient. 2012;11(2):127-31.

14. Santos Neto ET, Oliveira AE, Zandonade E, Leal MC. Acesso à assistência odontológica no acompanhamento pré-natal. Cienc saúde coletiva. 2012;17(11):3057-68.

15. Martins LO, Pinheiro RPS, Arantes DC, Nascimento LS, Santos Júnio PB. Assistência odontológica à gestante: 
percepção do cirurgião-dentista. Rev Pan-Amaz Saúde. 2013;4(4):11-18.

16. Jannotti CB, Leal N. Saúde bucal da gestante atendida pelo SUS: práticas e representaçóes de profissionais e pacientes. FEMINA. 2009;37(8):413-21.

17. Prestes ACG, Martins AB, Neves M, Mayer RTR. Saúde bucal materno-infantil: uma revisão integrativa. RFO. 2013;18(1):112-9.

18. Brasil. Ministério da Saúde. Secretaria de Atenção à Saúde. Departamento de Atenção Básica. Cadernos de Atenção Básica - nº17. Brasília: Ministério da Saúde; 2006.

19. Bastos RDS, Cardoso JÁ, de Farias JG, Falcão GGVCS. Desmistificando o atendimento odontológico à gestante. Revisão da literatura. Rev Bahiana de Odontol. 2014;5(2):104-116.

20. Brasil. Ministério da Saúde. Nota técnica: Rede Cegonha. Brasília: Ministério da Saúde; 2011.

21. Moimaz SAS, Rocha NB, Garbin CAS. O acesso de gestantes ao tratamento odontológico. Rev odontol Univ Cid São Paulo. 2007;19:39-45.
22. Vieira GF, Zocratto KBF. Percepcão das gestantes quanto a sua saúde bucal. RFO. 2007;12(2):27-31.

23. Ebrahim ZF, Oliveira MCQ, Peres MPSM, Franco JB. Tratamento odontológico em gestantes. Science in Health. 2014;5(1):32-44.

24. Marin C, Segura-Egea JJ, Martínez-Sahuquillo Á, Bullón P. Correlation between infant birth weight and mother's periodontal status. J Clin Periodontol. 2005;32(3):299-304.

25. Geisinger ML, Geurs NC, Bain JL, Kaur M, Vassilopoulos PJ, Cliver SP, Hauth JC, Reddy MS. Oral health education and therapy reduces gingivitis during pregnancy. J Clin Periodontol. 2014;41:141-8.

26. Jeffcoat, M, Parry S, Sammel M, Clothier B, Caitlin A, Macones, G. Periodontal infection and preterm birth: successful periodontal therapy reduces the risk of preterm birth. BJOG. 2011;118(2):250-6.

27. Berkowitz RJ. Acquisition and transmission of mutans streptococci. J Calif Dent Assoc. 2003;31(2):135-8.

\section{Como citar este artigo:}

Marín C, Maçaneiro CAR, Bottan ER, Vavassori F. Percepção do atendimento odontológico: comparações entre grupos de gestantes adultas e adolescentes. Rev. Aten. Saúde. 2015;13(46):65-71 